\title{
ELAPRENDIZAJE A TRAVÉS DEL FORO SEGÚN LOS ESTUDIANTES UNIVERSITARIOS. ESTUDIO DE CASO
}

\section{STUDENTS' PERCEPTION OF LEARNING FORUMS. CASE STUDY}

\author{
Dra. María Ángeles Andreu Andrés ${ }^{1}$ \\ maandreu@idm.upv.es \\ Miguel García Casas² \\ mgcasas5@yahoo.es
}

\begin{abstract}
${ }^{1}$ Universitat Politècnica de València.ETS Arquitectura, Departamento de Lingüística Aplicada. Canimo de vera, s/n 46022 Valencia (España)

${ }^{2}$ IES La Morería. Departamento de Biología. País Valenciano, 146920 Mislata-Valencia (España)
\end{abstract}

Este trabajo forma parte de una investigación mayor que quiere medir el progreso de la competencia comunicativa de 83 estudiantes de Grado a través de las TIC y, en concreto, del foro académico de su asignatura. En esta fase del estudio se analiza cuantitativamente la existencia de relaciones entre el uso que los estudiantes hacen de las TIC y sus percepciones de utilidad y aprendizaje, recogidas a través de un cuestionario al finalizar el último foro. De acuerdo con los resultados, no puede generalizarse que deseen incorporar las TIC como instrumentos de aprendizaje, ni que sus habilidades tecnológicas beneficien ese proceso de aprendizaje.

Palabras clave: TIC, foro, aprendizaje colaborativo, lengua extranjera.

This paper forms part of a broader research that aims to measure the communication skill development skills of 83 undergraduates' by using ICT and, particularly learning forums, for this subject. In this stage of the research, we did a quantitative analysis of the relationship between ICT, expected usefulness and learning processes. To collect the data, we used a questionnaire which was distributed at the end of the last forum. According to the results, it cannot be generalized that students look forward to incorporating ICT as a learning tool or the fact that their technological skills benefit from this learning process.

Keywords: ICT, learning forum, collaborative learning, foreign language. 


\section{Introducción.}

Desde la Declaración de Bolonia en 1999 y la implantación del Espacio Europeo de Educación Superior con un modelo de educación basado en competencias, cada día resulta más evidente la necesidad de coordinar las competencias adquiridas por los estudiantes a lo largo de sus estudios y las necesidades laborales de estos futuros profesionales. De acuerdo con Passow (2012), entre las competencias que más valoran los graduados al enfrentarse a su profesión se encuentran la competencia digital y la comunicativa en lengua extranjera y materna; unas competencias que, como indican en su estudio Torres-Coronas y Vidal-Blasco (2015), el mercado laboral les demanda independientemente de su titulación y están relacionadas con el uso del procesador de textos, la búsqueda en internet, el uso del correo electrónico y la colaboración en línea.

Desde el punto de vista educativo, las tecnologías de la información y la comunicación (TIC) amplían la oferta formativa, ofrecen entornos y escenarios educativos adicionales a los que tradicionalmente se vienen usando en el aula (Domingo \& Marqués, 2011) y potencian la interactividad y flexibilidad en la enseñanzaaprendizaje (Cabero, 2006). Asimismo, permiten incrementar las posibilidades para la orientación del alumnado, facilitan su autorización mientras favorecen el trabajo colaborativo y el autoaprendizaje.

Sin embargo, hemos de ser conscientes de que por sí mismas no ofrecen garantía de ningún cambio positivo en la enseñanza sino se cuenta con la activa participación y motivación de docentes y discentes además de un respaldo institucional que ofrezca formación adecuada a su profesorado; de modo que se evite la paradoja de encontrar docentes que se crean capacitados para utilizarlas TIC (Cantón \& Baelo, 2011), pero que desconozcan cómo integrarlas en su docencia (Area, 2010).

Los estudiantes universitarios de hoy en día, independientemente de que se les considere nativos digitales (Prensky,2001), estudiantes digitales (Gallardo, 2012), generación net (Taspscott, 1998; Oblinger \& Oblinger, 2005) o $i$-Generation (Rosen, 2010), entre otros términos, por lo general empezaron a utilizar ordenadores en su niñez y han estado siempre rodeados de teléfonos móviles, videojuegos o internet. Basándose en la hipótesis de que los actuales estudiantes han tenido una educación digital uniforme (Kennedy, Judd, Churchwards, Gray \& Krause, 2008; Jones, Ramanau, Cross \& Healing, 2010), se dice de ellos que procesan la información de manera distinta a los universitarios de décadas anteriores: suelen leer directamente de la pantalla, consultan antes internet que un libro, están acostumbrados a recibir información de manera rápida y las redes sociales parecen formar parte de sus vidas de manera habitual.

A pesar de todo ello partimos de la hipótesis de que no puede asumirse per se que estos estudiantes quieran incorporar las tecnologías que habitualmente usan en su rutina diaria como instrumentos de aprendizaje, ni que estas habilidades que despliegan cotidianamente puedan necesariamente beneficiar un proceso de aprendizaje basado en la tecnología.

Teniendo en cuenta las reflexiones de González, Espuny y Gisbert (2010) sobre el nivel de competencia digital de los graduados españoles y su incorporación a los currículos, entendemos que debemos, en primer lugar, ahondar en el uso que nuestros alumnos 
hacen de la tecnología dentro y fuera del aula universitaria para analizar tanto sus percepciones como la eficacia de esas tecnologías como herramientas de enseñanza-aprendizaje y de desarrollo competencial, de modo que podamos ofrecerles herramientas y entornos de aprendizaje adecuados.

\subsection{El foro académico.}

Sin inventar nada nuevo, es necesario incorporar al aula universitaria -junto con las técnicas y estrategias activas más acordes con los objetivos de enseñanza-aprendizaje que se persigan en la asignatura-aquellas herramientas tecnológicas que les son tan familiares (Laurillard, 2002). Tal es el caso del foro y, en particular, del foro académico (Andreu-Andrés \& Labrador-Piquer, 2010) que, como medio de comunicación asíncrona, puede servir para debatir temas de interés relacionados con sus estudios junto con contenidos de la asignatura; en él las aportaciones de cada estudiante quedan registradas y todos tienen acceso a ellas.

Entre sus beneficios podemos encontrar unas discusiones de mayor calidad (Callan, 2006; O'Neill, Duplock \& Willis, 2006) por el hecho de que las aportaciones puedan leerse con tiempo, como comunicación asíncrona que es (Karsenti, Collin\& Lira, 2012), lo que facilita la reflexión y la búsqueda de información por parte del estudiante antes de contestar a un hilo o conversación. Gracias a la herramienta tecnológica, se facilita el intercambio intelectual y la autonomía en el aprendizaje (Manprit, 2011). Si bien no está exenta de desventajas como la inversión de tiempo necesaria para leer los mensajes de los demás, contestar y buscar información además de la falta de contacto físico entre compañeros; algo que creemos imprescindible en el aula universitaria.

No obstante, si a los estudiantes se les ofrece un marco en el que las discusiones a través del foro estén relacionadas con sus intereses y estudios, tal y como se ha hecho en esta investigación, la herramienta puede llegar a convertirse en un estimulo para mejorar las competencias comunicativas. $\mathrm{Si} \mathrm{a}$ ello se le une la retroalimentación del propio docente, el foro puede llegar a ampliar sus oportunidades de aprendizaje y mejora (Peterson \& Caverly, 2006; Palomar, 2009), además de su satisfacción y motivación (Russo \& Benson, 2005; Krish, 2011), al menos teóricamente. Estudios como los de Dexter (2010) y Álvarez, Espasa y Guash (2012, en Coll, Rochera, de Gisbert \& Díaz-Barriga, 2013) demuestran que al incluir sugerencias y preguntas en esa retroalimentación en lugar de simples correcciones, los estudiantes son capaces de mejorar sus textos.

\subsection{Objetivo del estudio.}

Este estudio forma parte de una investigación más amplia en la que se quiere medir el progreso de las competencias comunicativas en lengua extranjera tras la incorporación del foro al programa de la asignatura con estudiantes de segundo y tercero de grado en Arquitectura.

Tras un primer paso en el que se ha analizado el uso que estos estudiantes hacen de las tecnologías dentro y fuera del ambiente universitario (Andreu-Andrés, 2014), este trabajo se propone medir la existencia de relaciones entre el uso que los estudiantes hacen de las TIC y sus percepciones de utilidad, aprendizaje y progreso. Una tercera y última fase de la investigación analizará la expresión escrita de los participantes antes y 
después de haber utilizado el foro de la asignatura a lo largo de un curso académico, lo que permitirá estudiar la mejora de la competencia comunicativa escrita en lengua extranjera.

\section{Metodología.}

A lo largo de un año académico se abrieron, espaciados entre sí, tres foros híbridos según la taxonomía de Abawajy y Kim (2011). El tema central de cada foro estaba directamente relacionado con temas de su carrera trabajados en la asignatura. En ellos se les invitaba a opinar y aportar información y puntos de vista razonados en la lengua extranjera, como parte integral de las clases presenciales a través de la plataforma de teleformación de nuestra Universidad, PoliformaT. En la investigación participaron

\begin{tabular}{|l|}
\hline \multicolumn{1}{|c|}{ Puntúa cada declaración de $\mathbf{0 , 1}$ a 10} \\
$\mathbf{0 , 1 - 2 , 0}=$ Estoy totalmente desacuerdo \\
$\mathbf{4 , 1 - 6 , 0}=$ Ni estoy de acuerdo ni en desacuerdo \\
$\mathbf{6 , 1 - 8 , 0}=$ Estoy de acuerdo \\
$\mathbf{8 , 1 - 1 0}=$ Estoy en total acuerdo \\
\hline 1. $\quad$ Me gustan las redes sociales \\
\hline 2. Uso las redes sociales \\
\hline 3. $\quad$ La tecnología me ayuda a estar en contacto con mis amigos \\
\hline 4. $\quad$ Me gustan los ordenadores \\
\hline 5. $\quad$ No me gustan los ordenadores \\
\hline 6. La tecnología me ayuda en mis estudios en casa \\
\hline 7. $\quad$ La tecnología me ayuda en mis estudios en la universidad \\
\hline 8. La tecnología me ayudará en mi profesión \\
\hline 9. Uso la tecnología para entretenerme \\
\hline 10. Prefiero leer libros o revistas impresas \\
\hline 11. Prefiero leer libros electrónicos \\
\hline 12. Cuando necesito comprobar información uso internet \\
\hline 13. Las actividades colaborativas en línea me ayudan a aprender \\
\hline 14. Las actividades colaborativas en general son una pérdida de tiempo \\
\hline 15. Me gusta trabajar en grupo \\
\hline 16. Las actividades colaborativas cara a cara en clase me ayudan a aprender \\
\hline 17. El foro de la asignatura es útil para mejorar mi expresión escrita \\
\hline 18. El foro de la asignatura es una pérdida de tiempo \\
\hline 19. El foro de la asignatura es útil para promover mi pensamiento crítico \\
\hline 20. El foro de la asignatura es útil para mejorar mi comprensión lectora \\
\hline 21. El foro es útil para poner en práctica lo que voy aprendiendo en la asignatura \\
\hline 22. El foro es útil para intercambiar puntos de vista y gustos personales \\
\hline 23. El foro es útil para aprender de los demás compañeros \\
\hline 24. No creo que pueda aprender de los demás compañeros \\
\hline 25. Me gusta estar sólo \\
\hline 26. Tengo muchos amigos \\
\hline 27. Prefiero utilizar la tecnología para mejorar mi comprensión oral \\
\hline 28. Prefiero utilizar la tecnología para mejorar y repasar la gramática inglesa \\
\hline 29. Prefiero las clases tradicionales \\
\hline 30. No puedo estar sin mi móvil \\
\hline
\end{tabular}

Tabla 1. Declaraciones traducidas al español. Cuestionario no validado (Andreu-Andrés, 2014). 


$\begin{array}{ccc}\begin{array}{c}\text { Declaración } \\ \text { (S) }\end{array} & \text { Muestra } & \text { Media } \\ & & \\ \text { S1 } & 83 & 7,4 \\ \text { S2 } & 83 & 7,8 \\ \text { S3 } & 83 & 8,5 \\ \text { S4 } & 83 & 7,8 \\ \text { S5 } & 83 & 1,9 \\ \text { S6 } & 83 & 8,4 \\ \text { S7 } & 83 & 8,3 \\ \text { S8 } & 83 & 9,1 \\ \text { S9 } & 83 & 7,2 \\ \text { S10 } & 83 & 6,9 \\ \text { S11 } & 83 & 3,3 \\ \text { S12 } & 83 & 8 \\ \text { S13 } & 83 & 6,8 \\ \text { S14 } & 83 & 3,1 \\ \text { S15 } & 83 & 6,3 \\ \text { S16 } & 83 & 7,3 \\ \text { S17 } & 83 & 7,7 \\ \text { S18 } & 83 & 2,6 \\ \text { S19 } & 83 & 6 \\ \text { S20 } & 83 & 7 \\ \text { S21 } & 83 & 7,3 \\ \text { S22 } & 83 & 7,6 \\ \text { S23 } & 83 & 7,2 \\ \text { S24 } & 83 & 1,8 \\ \text { S25 } & 83 & 3,1 \\ \text { S26 } & 83 & 7,3 \\ \text { S27 } & 83 & 6,9 \\ \text { S28 } & 83 & 6,9 \\ \text { S29 } & 83 & 6,4 \\ \text { S30 } & 83 & 5,7\end{array}$

\begin{tabular}{|c|c|c|}
\hline $\begin{array}{c}\text { Desviación } \\
\text { estándar }\end{array}$ & $\begin{array}{l}\text { Puntuación } \\
\text { mínima } \\
\text { (entre 0,1 y } \\
10)\end{array}$ & $\begin{array}{c}\text { Puntuación } \\
\text { máxima } \\
\text { (entre 0,1 y } \\
\text { 10) }\end{array}$ \\
\hline 1,7 & 0,1 & 10 \\
\hline 1,8 & 2,1 & 10 \\
\hline 1,4 & 4 & 10 \\
\hline 1,6 & 4 & 10 \\
\hline 1,8 & 0,1 & 6 \\
\hline 1,3 & 5 & 10 \\
\hline 1,5 & 3 & 10 \\
\hline 0,8 & 7 & 10 \\
\hline 1,6 & 2,6 & 10 \\
\hline 2,4 & 1 & 10 \\
\hline 2,7 & 0,1 & 10 \\
\hline 1,5 & 4 & 10 \\
\hline 1,7 & 2 & 10 \\
\hline 1,9 & 0,1 & 8 \\
\hline 1,6 & 2,1 & 10 \\
\hline 1,9 & 0,1 & 10 \\
\hline 1,6 & 3 & 10 \\
\hline 1,9 & 0,1 & 8 \\
\hline 2,2 & 0,2 & 10 \\
\hline 1,7 & 2 & 10 \\
\hline 2,0 & 1 & 10 \\
\hline 1,7 & 2 & 10 \\
\hline 1,6 & 2 & 10 \\
\hline 1,7 & 0,1 & 7 \\
\hline 2,3 & 0,1 & 8 \\
\hline 1,5 & 0,1 & 10 \\
\hline 1,5 & 4 & 10 \\
\hline 1,5 & 2,1 & 10 \\
\hline 1,9 & 0,1 & 10 \\
\hline 2,6 & 0,1 & 10 \\
\hline
\end{tabular}

Tabla 2. Resumen estadístico (Andreu-Andrés, 2014).

83 estudiantes de segundo y tercero de Grado en Arquitectura con una media de edad de 20 años. Los tres foros fueron moderados por el profesor.

Al finalizar el último, y de manera anónima, los estudiantes puntuaron las treinta declaraciones que recoge la Tabla 1 , traducidas al español, como alternativa al cuestionario tipo Likert que pretende captar la diversidad y variabilidad en las respuestas; respuestas que fueron adelantadas en Andreu-Andrés (2014). A partir de ellas se han realizado los análisis estadísticos que conforman esta segunda fase de la investigación y que se recogen a partir de la Tabla 3. Las declaraciones han sido desarrolladas a partir de los cuestionarios de otros autores (Kennedy et al., 2008) y quedan recogidas en la Tabla 1 para facilitar la comprensión de los resultados.

\section{Resultados.}

La Tabla 2 ofrece el resumen estadístico de cada una de las declaraciones. Con objeto de estudiar la existencia o no de relaciones entre pares de estas variables (declaraciones) y su grado de relación, se realiza un análisis de correlaciones de Pearson. El rango de estos coeficientes de correlación va de $-1 \mathrm{a}+1$ y miden la fuerza de la relación lineal entre las variables; cuanto más cerca de estos valores 


\begin{tabular}{|c|c|}
\hline $\begin{array}{l}\text { A quienes les gustan las redes } \\
\text { sociales (S1) y las usan (S2)... }\end{array}$ & $\begin{array}{l}\text { les agradan los ordenadores (S4); consideran que la tecnología les } \\
\text { ayuda en los estudios en casa (S6), en la universidad (S7) y a estar en } \\
\text { contacto con sus amigos (S3). } \\
\text { Consultan información en internet (S12). Aseguran que pueden vivir } \\
\text { sin su móvil (S30) pero creen que no pueden aprender de los demás } \\
\text { compañeros (S24) y prefieren utilizar las tecnologías para mejorar su } \\
\text { competencia gramatical en lengua extranjera (S28). }\end{array}$ \\
\hline $\begin{array}{l}\text { Cuanto más creen que la } \\
\text { tecnología les permite estar en } \\
\text { contacto con los demás (S3)... }\end{array}$ & $\begin{array}{l}\text { más amigos aseguran tener (S26). Prefieren utilizar las TIC para } \\
\text { mejorar la comprensión oral (S27) y la gramática (S27) y sienten una } \\
\text { mayor dependencia del móvil (S30). }\end{array}$ \\
\hline $\begin{array}{l}\text { Cuanto más les gustan los } \\
\text { ordenadores (S4)... }\end{array}$ & $\begin{array}{l}\text { están más convencidos de que el foro es provechoso para aumentar } \\
\text { su expresión escrita (S17), poner en práctica lo aprendido en la } \\
\text { asignatura (S21), aprender de los demás (S23) y estar en contacto con } \\
\text { sus amistades (S3). }\end{array}$ \\
\hline $\begin{array}{l}\text { Cuanto } \\
\text { ordenac }\end{array}$ & $\begin{array}{l}\text { menos utilizan la tecnología para entretenerse (S9), más consideran } \\
\text { el foro una pérdida de tiempo (S18). Prefieren leer libros o revistas } \\
\text { impresas (S10). }\end{array}$ \\
\hline $\begin{array}{l}\text { Quienes consideran que la } \\
\text { tecnología les ayuda en sus } \\
\text { estudios en casa (S6) y en la } \\
\text { universidad (S7)... }\end{array}$ & $\begin{array}{l}\text { afirman que también les ayudará en su profesión (S8). Sin embargo, } \\
\text { prefieren utilizar la tecnología para mejorar la comprensión oral } \\
\text { (S27) y la gramática (S28) en la clase de lengua extranjera. }\end{array}$ \\
\hline $\begin{array}{l}\text { Quienes consideran que la } \\
\text { tecnología les ayudará en su } \\
\text { profesión }(\mathrm{S} 8) \ldots\end{array}$ & $\begin{array}{l}\text { encuentran mayor utilidad al foro para intercambiar pareceres (S22), } \\
\text { mejorar la expresión escrita (S17) y aprender de los demás (S23). Sin } \\
\text { embargo, prefieren utilizar la tecnología para mejorar su } \\
\text { competencia gramatical en la clase de lengua extranjera (S28) }\end{array}$ \\
\hline $\begin{array}{l}\text { in la tecnología para } \\
9) \text {... }\end{array}$ & $\begin{array}{l}\text { afirman que les gustan los ordenadores (S4); la tecnología les permite } \\
\text { estar en contacto con sus amistades (S3) y el foro lo consideran útil } \\
\text { para mejorar la expresión escrita (S17). }\end{array}$ \\
\hline $\begin{array}{l}\text { Quien } \\
\text { electró }\end{array}$ & $\begin{array}{l}\text { consideran que el foro ayuda a aprender de los demás (S23), a poner } \\
\text { en práctica lo aprendido en la asignatura (S21) y a promover el } \\
\text { pensamiento crítico (S19). }\end{array}$ \\
\hline $\begin{array}{l}\text { Quienes usan internet cuando } \\
\text { precisan consultar información } \\
\text { (S12)... }\end{array}$ & $\begin{array}{l}\text { creen que las actividades colaborativas en línea les ayudan a aprender } \\
\text { (S13) y reconocen que no pueden vivir sin su teléfono móvil (S30). }\end{array}$ \\
\hline $\begin{array}{l}\text { Para quienes las actividades } \\
\text { colaborativas de aula ayudan a } \\
\text { aprender }(\mathrm{S} 16) \ldots\end{array}$ & $\begin{array}{l}\text { el foro les resulta útil para mejorar la expresión escrita (S17) y la } \\
\text { comprensión lectora (S20). }\end{array}$ \\
\hline $\begin{array}{l}\text { Para quienes las actividades } \\
\text { grupales en línea ayudan a } \\
\text { aprender }(\mathrm{S} 13) \ldots\end{array}$ & $\begin{array}{l}\text { el foro resulta útil para mejorar la expresión escrita (S17), la } \\
\text { comprensión lectora (S20) así como para aprender de sus pares (S23) } \\
\text { e intercambiar puntos de vista (S22). }\end{array}$ \\
\hline $\begin{array}{l}\text { Los que creen que el foro ayuda a } \\
\text { promover el pensamiento crítico } \\
\text { (S19)... }\end{array}$ & $\begin{array}{l}\text { opinan que les ayuda también a mejorar la expresión escrita (S17), la } \\
\text { comprensión lectora (S20), a intercambiar puntos de vista (S22), } \\
\text { poner en práctica lo aprendido (S21) y aprender de los demás (S23). }\end{array}$ \\
\hline $\begin{array}{l}\text { Cuanto más defienden la utilidad } \\
\text { del foro para poner en práctica lo } \\
\text { aprendido en la asignatura }(\mathrm{S} 21) \text {.. }\end{array}$ & $\begin{array}{l}\text { encuentran una mayor utilidad a éste para intercambiar opiniones } \\
\text { (S22) y aprender de los demás (S23). }\end{array}$ \\
\hline $\begin{array}{l}\text { Quienes consideran que el foro } \\
\text { contribuye a mejorar su expresión } \\
\text { escrita (S17) y la comprensión } \\
\text { lectora (S20)... }\end{array}$ & $\begin{array}{l}\text { creen que también les sirve para poner en práctica lo aprendido en la } \\
\text { asignatura (S21), a intercambiar puntos de vista (S22) y aprender de } \\
\text { los demás (S23). Curiosamente todos afirman que les gusta la soledad } \\
\text { (S25). }\end{array}$ \\
\hline $\begin{array}{l}\text { Cuanto mayor es la defensa del } \\
\text { foro para el intercambio de puntos } \\
\text { de vista }(\mathrm{S} 22) \ldots\end{array}$ & $\begin{array}{l}\text { mayor es la creencia de que el foro les sirve para aprender de los } \\
\text { demás (S23) y viceversa. }\end{array}$ \\
\hline
\end{tabular}

Tabla 3. Correlaciones directas entre variables. 


\begin{tabular}{|c|c|}
\hline $\begin{array}{l}\text { Cuanto más les agradan las redes } \\
\text { sociales (S1)... }\end{array}$ & menos prefieren las clases tradicionales (S29). \\
\hline $\begin{array}{l}\text { Cuanto más les desagradan los } \\
\text { ordenadores (S5)... }\end{array}$ & $\begin{array}{l}\text { menos les gustan las redes sociales (S1) y los libros } \\
\text { electrónicos (S11); en menor medida usan internet } \\
\text { para consultar información (S12), menos consideran } \\
\text { que los ordenadores les ayuden en sus estudios en la } \\
\text { universidad (S7) o en su futura profesión (S8) y que } \\
\text { el foro les sirva para intercambiar pareceres (S22). Su } \\
\text { preferencia por la tecnología para mejorar sus } \\
\text { conocimientos de gramática (S28) es menor. }\end{array}$ \\
\hline $\begin{array}{l}\text { Cuanto más señalan que la tecnología } \\
\text { les resulta útil para estudiar en casa } \\
\text { (S6)... }\end{array}$ & menos les gusta trabajar en grupo (S15). \\
\hline $\begin{array}{l}\text { Cuanto más afirman que la tecnología } \\
\text { les resulta útil en sus estudios en la } \\
\text { universidad (S7)... }\end{array}$ & menos les gusta trabajar en grupo (S15). \\
\hline $\begin{array}{l}\text { Cuanto más consideran que la } \\
\text { tecnología les ayudará en su profesión } \\
\text { (S8)... }\end{array}$ & $\begin{array}{l}\text { se sienten menos dependientes del teléfono móvil } \\
\text { (S30). }\end{array}$ \\
\hline $\begin{array}{l}\text { Cuanto más prefieren los libros } \\
\text { impresos }(\mathrm{S} 10) \ldots\end{array}$ & $\begin{array}{l}\text { menos les gustan y usan las redes sociales (S1 y S2), } \\
\text { menos consultan información en internet (S12). }\end{array}$ \\
\hline $\begin{array}{l}\text { lás usan internet para obtener } \\
\text { ón }(\mathrm{S} 12) \ldots\end{array}$ & $\begin{array}{l}\text { menos les gusta estar solos (S25) y prefieren en menor } \\
\text { medida las clases tradicionales (S29). }\end{array}$ \\
\hline $\begin{array}{l}\text { Cuanto más apoyan las actividades } \\
\text { grupales en línea }(\mathrm{S} 13) \ldots\end{array}$ & $\begin{array}{l}\text { menos consideran el foro una pérdida de tiempo } \\
\text { (S18), en menor medida disfrutan de la soledad (S25) } \\
\text { y de los libros impresos (S10), }\end{array}$ \\
\hline $\begin{array}{l}\text { Cuanto más consideran una pérdida de } \\
\text { tiempo las actividades colaborativas en } \\
\text { general }(\mathrm{S} 14) \ldots\end{array}$ & $\begin{array}{l}\text { menos creen que las actividades grupales de aula les } \\
\text { ayuden a aprender (S16), que el uso del foro favorezca } \\
\text { la expresión escrita (S17), que mejore la comprensión } \\
\text { lectora (S20) o permita poner en práctica lo aprendido } \\
\text { en la asignatura (S21)++ }\end{array}$ \\
\hline $\begin{array}{l}\text { Cuanto más les gusta trabajar en equipo } \\
\text { en el aula }(S 15) \ldots\end{array}$ & menos atractivas les resultan las redes sociales (S1) \\
\hline $\begin{array}{l}\text { ldan las actividades } \\
1 \text { aula }(\mathrm{S} 16) \ldots\end{array}$ & $\begin{array}{l}\text { menos consideran el foro una pérdida de tiempo } \\
\text { (S18). Creen que la tecnología les ayudará en su } \\
\text { profesión (S8) pero prefieren los libros o revistas } \\
\text { impresas (S10). }\end{array}$ \\
\hline $\begin{array}{l}\text { Cuanto más consideran el foro una } \\
\text { pérdida de tiempo (S18)... }\end{array}$ & $\begin{array}{l}\text { menos creen que la tecnología les ayude en sus } \\
\text { estudios (S7) y en su profesión (S8). Menor utilidad } \\
\text { le encuentran al foro para mejorar la expresión escrita } \\
\text { (S17), la comprensión lectora (S20), el intercambio de } \\
\text { puntos de vista (S22) o para aprender de los demás } \\
\text { (S23). }\end{array}$ \\
\hline $\begin{array}{l}\text { Cuanto más defienden la idea de que no } \\
\text { pueden aprender de sus pares (S24)... }\end{array}$ & $\begin{array}{l}\text { entienden que el foro no ayuda a fomentar el espíritu } \\
\text { crítico (S19) y la comprensión lectora (S20); } \\
\text { consideran, en menor medida, que la tecnología les } \\
\text { ayuda en sus estudios en casa (S6). Afirman en mayor } \\
\text { medida que les gusta la soledad (S25) pero defienden } \\
\text { menos las clases tradicionales (S29). }\end{array}$ \\
\hline $\begin{array}{l}\text { Cuanto más prefieren utilizar la } \\
\text { tecnología para mejorar su comprensión } \\
\text { oral (S27), como habitualmente hacen } \\
\text { en el aula de lengua,... }\end{array}$ & $\begin{array}{l}\text { menor utilidad le encuentran al foro para mejorar la } \\
\text { comprensión lectora (S20) a través de los mensajes de } \\
\text { sus pares. }\end{array}$ \\
\hline $\begin{array}{l}\text { Cuanto mayor es su dependencia del } \\
\text { teléfono móvil (S30)... }\end{array}$ & $\begin{array}{l}\text { menos entienden que el foro les ayude a mejorar la } \\
\text { expresión escrita (S17). }\end{array}$ \\
\hline
\end{tabular}

Tabla 4. Correlaciones inversas entre variables. 


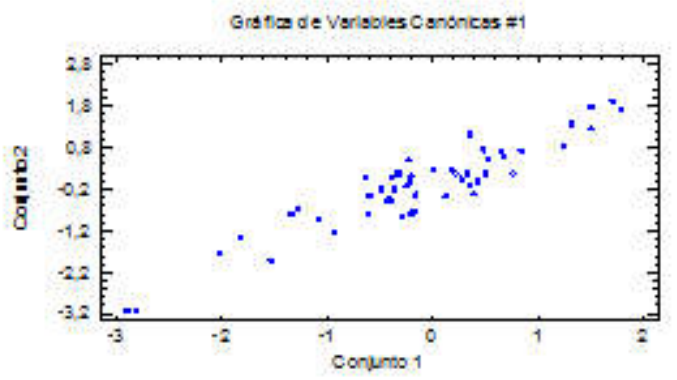

Figura 1. Análisis canónico

\begin{tabular}{|c|c|ccc}
$\begin{array}{c}\text { NÚMERO DE } \\
\text { CORRELACIÓN }\end{array}$ & $\begin{array}{c}\text { CORRELACIÓN } \\
\text { CANÓNICA }\end{array}$ & $\begin{array}{c}\text { CHI } \\
\text { CUADRADO }\end{array}$ & $\begin{array}{c}\text { GRADOS } \\
\text { DE } \\
\text { LIBERTAD }\end{array}$ & $\begin{array}{c}\text { NIVEL DE } \\
\text { CONFIANZA } \\
(\boldsymbol{\%})\end{array}$ \\
$\mathbf{1}$ & 0,9306 & 598,391 & 225 & 99,99 \\
$\mathbf{2}$ & 0,8929 & 464,719 & 196 & 99,99 \\
$\mathbf{3}$ & 0,8732 & 358,537 & 169 & 99,99 \\
$\mathbf{4}$ & 0,7976 & 262,922 & 144 & 99,99 \\
$\mathbf{5}$ & 0,7377 & 195,672 & 121 & 99,99 \\
$\mathbf{6}$ & 0,7081 & 143,421 & 100 & 99,71 \\
\hline
\end{tabular}

Tabla 6. Resultados de la correlación canónica

se encuentren, mayor será la relación entre las variables.

Detectar la existencia de relaciones y su grado de intensidad puede dar pistas sobre el uso que el alumnado hace de la tecnología y la utilidad que le encuentra al foro para la enseñanza-aprendizaje de lengua extranjera. Para mayor claridad de los resultados, la Tabla 5 (véase Anexo) recoge sólo las relaciones significativas entre variables con un nivel de confianza de al menos el 95\%, en total 139 casos. De entre todas ellas, las Tablas 3 y 4 ofrecen de izquierda a derecha las correlaciones directas e inversas, respectivamente, que se consideran de mayor relevancia para nuestro objeto de estudio.

Seguidamente, la Tabla 4 ofrece, de izquierda a derecha, las correlaciones inversas más significativas de entre todas las que figuran en la Tabla 5 (véase Anexo).

Teniendo en cuenta la gran cantidad de relaciones estadísticamente significativas entre pares de variables (139 casos) y la difícil interpretación de todas ellas en su conjunto, se ha querido afinar un poco más el panorama que dichas correlaciones ofrecen a través del cálculo de las correlaciones canónicas entre dos conjuntos de 15 variables cada uno; por una parte, el formado por las variables que se refieren a los gustos de los informantes, a sus preferencias y acciones (S1, S4, S5, S10, S11, S15, S25, S27, S28, S29, S30, S2, S9, S12 y $\mathrm{S} 26) \mathrm{y}$, por otra, el relacionado con sus opiniones (S3, S6, S7, S8, S13, S14, S16, S17, S18, S19, S20, S21, S22, S23 y S24). 
Gráfica de Pesos del Componente

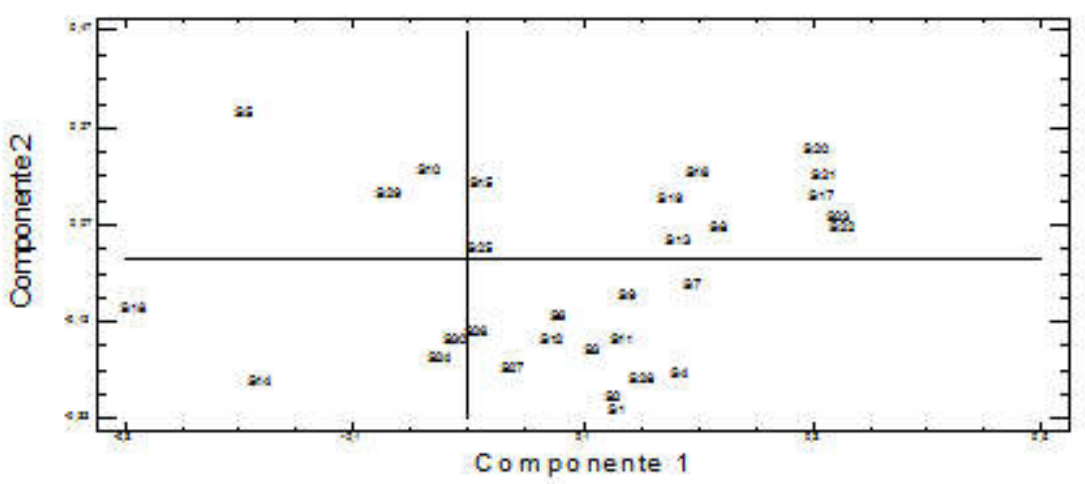

Figura2. Análisis de componentes principales

Los resultados de la correlación canónica muestran seis posibles combinaciones de estos dos conjuntos de variables que responden a relaciones estadísticamente significativas. Por tanto, se puede afirmar que estamos ante un conjunto estructurado y dependiente de variables.

Puede observarse en la Figura 1 el fuerte ajuste a una recta que expresaría la máxima correlación posible. En ella se aprecia un punto para cada encuesta contestada por los informantes; los ejes X e Y representan los dos grupos de 15 variables para la correlación canónica más significativa de entre las seis encontradas, con un valor de 0,9306 (véase Tabla 6).

En este caso, el análisis canónico permite expresar la fortaleza de la relación pero no las claves de su naturaleza, de ahí que se realice un análisis de componentes principales para intentar obtener un gráfico en el que se muestre, de modo visual, la relación entre esas variables sobre un espacio determinado por los dos primeros componentes (véase Figura $2)$. Este proceso matemático permite representar en pocas dimensiones o componentes una realidad multidimensional, lo que posibilita reducir la dimensionalidad de la matriz de los datos a fin de evitar redundancias y destacar relaciones, tal y como señalan González, De Pascual, Torres, y Garnica (2007).

El análisis descubre varios componentes entre los que el primero y el segundo recogen la mayor parte de la variabilidad: el 31,95\% de la varianza (el primer componente explica el $19,05 \%$ y el segundo el $12,90 \%$ de la varianza) y ofrece una imagen interesante aunque algo borrosa dado que los dos primeros componentes explican una moderada cantidad de la varianza.

Las proximidades entre los puntos (variables) indican el grado de correlación que existe entre ellas; así a la izquierda del gráfico, la variable S5 («No me gustan los ordenadores») y dos variables relacionadas con ella, S10 («Prefiero leer libros o revistas impresas») y S29 («Prefiero las clases tradicionales»), se encuentran junto con las 
S18, S14 y S24 relacionadas con la inutilidad del foro («El foro de la asignatura es una pérdida de tiempo», «Las actividades colaborativas en general son una pérdida de tiempo» $\mathrm{y}$ «No creo que pueda aprender de los demás»).

En el otro extremo del espectro (lado derecho del gráfico), se encuentran las variables que están directamente relacionadas con el gusto por los ordenadores y el uso de las redes sociales (S1, S2, S3, S4, S6, S9, S11, S12), así como a favor de la utilidad del foro (S17, S19, S20, S21, S22, S23) y de las actividades colaborativas, tanto en línea como en el aula (S13, S16).

\section{Conclusiones.}

Tras un primer estudio en el que se analizó el uso que los estudiantes que han participado en la experiencia hacen de las tecnologías dentro y fuera del ambiente universitario (Andreu-Andrés, 2014), esta segunda fase de la investigación ha medido la existencia de relaciones entre ese uso de las TIC y sus percepciones de utilidad, aprendizaje y progreso al emplearlas; se trata de un paso previo a la fase final de la investigación en la que se medirá la mejora real en la expresión escrita de los participantes tras el uso del foro como medio de comunicación asíncrona para debatir temas de interés relacionados con su titulación y los contenidos de la asignatura. Una herramienta que consideramos fomenta el espíritu crítico y la colaboración, además de ayudar a mejorar la expresión y comprensión escrita en lengua extranjera.

Se ha probado la existencia de relaciones estadísticamente significativas entre pares de variables calculando los coeficientes de correlación entre ellas. En un total de 139 casos los coeficientes de correlación son estadísticamente significativos. El análisis canónico ratifica esas relaciones mientras que el análisis de componentes principales confirma, de manera visual, los resultados obtenidos en el análisis de correlaciones. Puede afirmarse, por tanto, la existencia de relaciones entre el uso tecnológico que hacen los estudiantes y sus percepciones de utilidad y aprendizaje a través del foro académico. De acuerdo con los resultados se pueden distinguir varios grupos de participantes en función de su relación con las TIC; se muestran en cuatro subapartados dentro de las conclusiones -a partir de las correlaciones directas e inversas que recogen las Tablas 3 y 4 - para facilitar su lectura.

\subsection{Gusto por los ordenadores.}

Los análisis distinguen dos grandes grupos: aquellos que reconocen que no les gustan los ordenadores de a quienes sí les agradan. Los primeros son defensores de las clases tradicionales y de los libros o revistas impresas, consideran el foro una herramienta inútil, probablemente porque tampoco creen que puedan aprender de sus pares.

Por contra, a los segundos no sólo les agradan los ordenadores, sino que usan las redes sociales y opinan que la tecnología les sirve para estar en contacto con sus amistades, obtener información y entretenerse. Prefieren los libros y revistas electrónicas y defienden las actividades colaborativas tanto en el aula como en línea. Asimismo, consideran que el foro es provechoso para mejorar la expresión escrita, promover el pensamiento crítico, mejorar la comprensión lectora, poner en práctica lo que van aprendiendo, intercambiar opiniones y, en definitiva, aprender con y de los demás. 
Unas afirmaciones que son más patentes cuanto mayor es su gusto por la tecnología.

Sin embargo, a quienes no les gustan los ordenadores pero prefieren utilizar esa tecnología para mejorar sus conocimientos de gramática en la clase de lengua, el foro resulta poco provechoso para mejorar la comprensión lectora. Por otra parte, cuanto más dependientes son del teléfono móvil, menos entienden que el foro les pueda ayudar a mejorar la expresión escrita; un sentimiento que no sabemos si tendrá su origen en la diferencia entre el tipo de escritura que suelen utilizar cuando envían o reciben mensajes de móvil o whatsApp y las reglas acordadas para la participación en el foro.

\subsection{Gusto por las actividades colaborativas.}

De igual modo puede hacerse una distinción entre los defensores de las actividades colaborativas en el aula o en línea y aquéllos para quienes resulta una pérdida de tiempo. Como quizás cabría esperar, si los primeros consideran que el foro les ayuda a mejorar sus destrezas comunicativas, los segundos defienden todo lo contrario. Quienes opinan que no es posible aprender de los compañeros, enfatizan la inutilidad del foro para mejorar la comprensión lectora, probablemente porque no hayan utilizado el foro para opinar y menos aún para leer los mensajes de los demás al creer que poco o nada pueden aprender de sus pares.

Por contra, quienes consideran que la tecnología les ayuda en sus estudios tanto en casa como en la universidad, creen que también lo hará en su profesión; el foro les resulta útil para intercambiar gustos $\mathrm{y}$ opiniones, aprender de los demás y mejorar la expresión escrita. En una clase de lengua extranjera prefieren usar la tecnología para mejorar la comprensión oral y la gramática, probablemente por estar habituados a hacerlo en la asignatura.

Como también parece lógico, el foro resulta inútil para quienes entienden que la tecnología les ayuda poco en sus estudios y que no lo hará demasiado en su profesión. Llama la atención el hecho de que cuanto más recalcan estos alumnos que usan la tecnología y consideran que ésta les es útil para sus estudios, tanto en la universidad como en casa, en menor medida defienden el trabajo en grupo, especialmente en una titulación que promueve el trabajo en equipo en gran parte de sus asignaturas. Se trata, en nuestra opinión, de un hecho en el que debería profundizarse a fin de encontrar los motivos que les hacen optar por esta postura ante una profesión en la que, necesariamente, tendrán que poner en práctica esa competencia transversal.

En una línea similar, los análisis descubren que cuanto mayor es el rechazo de los estudiantes por las actividades colaborativas en general -al considerarlas una pérdida de tiempo y, por ende, se entiende que prefieren el trabajo individual -, en menor medida consideran que las actividades grupales en el aula les ayuden a aprender, ni que el foro les permita mejorar su expresión escrita y comprensión lectora en lengua extranjera o, siquiera, poner en práctica lo aprendido en la asignatura. Por el contrario, y como sería de esperar ante estos resultados, cuanto más respaldan las actividades colaborativas en el aula y el trabajo en grupo, mayor en su defensa de la tecnología y del foro, si bien prefieren el libro o la revista impresa frente a la electrónica o digital. 


\subsection{Gusto por la soledad.}

Por último, los análisis muestran un nuevo grupo: aquellos a quienes les gusta la soledad y para los que el foro resulta ser una herramienta que contribuye a mejorar la expresión escrita, poner en práctica lo aprendido y, en definitiva, compartir puntos de vista y aprender de los demás. Unos resultados que nos hacen pensar en otra variable a tener en cuenta a la hora de utilizar la herramienta: la personalidad del discente, a fin de poder ofrecerle entornos de aprendizaje en los que se sienta motivado para aprender.

\subsection{En definitiva.}

De acuerdo con los resultados, y tal y como asumíamos en nuestra hipótesis inicial, no puede generalizarse que a estos estudiantes digitales (Gallardo, 2012) les gusten y deseen, necesariamente, incorporar las tecnologías que habitualmente usan en su rutina diaria como instrumentos de aprendizaje, ni que estas habilidades que despliegan cotidianamente puedan necesariamente beneficiar un proceso de aprendizaje basado en la tecnología.

Se trata de unos resultados que no podemos contrastar por no haber encontrado estudios similares al nuestro pero están en línea, por una parte, con los obtenidos por autores como Kennedy et al. (2008) cuando se cuestionan si todos los estudiantes de ahora son nativos digitales reales y, por otra, con los de Aileen (2008) cuando afirma que el foro puede proporcionar autenticidad y refuerzo de la comunicación escrita, junto con una mejora de la comprensión lectora -como recalca Schuetze (2010) - al despertar el interés por leer las contribuciones de los compañeros. Una utilidad que los propios alumnos perciben en mayor o menor medida, de acuerdo con las variables estudiadas.

Teniendo en cuenta todo lo anterior y los objetivos del proyecto de investigación en su conjunto, queda para una tercera fase del estudio medir el progreso de las competencias comunicativas de los participantes tras el uso de la herramienta tecnológica incorporada al programa de la asignatura.

Confiamos en que los resultados de esa tercera etapa, junto a las percepciones de utilidad y progreso de la herramienta obtenidas en esta segunda, ofrezcan una visión de la realidad más cercana; lo que, en nuestra opinión, posibilitaría el diseño de entornos de aprendizaje en los que las TIC tuvieran un protagonismo más ajustado a esa realidad y a los objetivos docentes que se persigan en la asignatura.

\section{Referencias bibliográficas.}

Abawajy, J. \& Kim, T. (2011). Online Learning Environment: Taxonomy of Asynchronous Online Discussion Forums. Software Engineering, Business Continuity, and Education Communications in Computer and Information Science, 257, 706714.

Aileen, Ng. (2008). Using Discussion Forums for ESL Communication Skills. TheInternetTESL Journal, 15 (10). Recuperado de http://iteslj.org/Techniques/ Ng-DiscussionForums.html

Álvarez, I., Espasa, A. \& Guasch, T. (2012). The value of feedback in improving collaborative writing assignments in an online learning environment. Studies in Higher Education, 37 (4),387-400. doi:10.1080/ 03075079.2010.510182 
Andreu-Andrés, Ma . Á. (2014). El foro académico y los estudiantes de lengua extranjera de especialidad. En F. Olmo \& J.M. Mangiante (Eds.). II Coloquio francoespañol de análisis del discurso y enseñanza de lenguas para fines específicos (pp. 189196). Valencia: Editorial UPV

Andreu-Andrés, Mª́.A \&abrador-Piquer, Ma.J. (2010). Técnica del puzle de aronson implementada con recursos electrónicos. 9 th International Conference of AELFE (pp. 112). Hamburg: Universität Hamburg, Institut für Germanistik - CD-Rom.

Area, M. (2010). El proceso de integración y uso pedagógico de las TIC en los centros educativos. Un estudio de casos. Revista de Educación, 325, 77-97.Recuperado de http:/ /www.revistaeducacion.educacion.es/re352/ re352.pdf

Cabero, J. (2006). Bases pedagógicas para la integración de las TIC en Primaria y Secundaria. II Congreso Internacional UNIVER. Universidad de Sevilla: Grupo de Tecnología Educativa de la Universidad de Sevilla. Recuperado de http://tecnologiaedu .us.es/cuestionario/bibliovir/Bases456.pdf

Callan, D. (2006). Pros and cons of having a discussion forum. Internet Marketing. Recuperado de http://www.akamarketing. com/forums-pros-and-cons.html

Cantón, I. \& Baelo, R. (2011). El profesorado universitario y las tecnologías de la información y la comunicación (TIC): disponibilidad y formación. Educatio Siglo XXI, 29 (1), 263-302.

Coll, C., Rochera, M., De Gispert, I. \& DíazBarriga, F. (2013). Distribution of feedback among teacher and students in online collaborative learning in small groups. Digital Review, 23, 27-46. Recuperado de http:// revistes.ub.edu/index.php/der/article/view/ $11285 / p d f$
Dexter, S. (2010). E-feedback intersections and disconnections in the interests of designers and users. International Journal of Engineering Education and life-long learning, 20 (2), 169-188.doi: 10.1504/ IJCEELL.2010.036814

Domingo, M. \& Marqués, P. (2011). Aulas 2.0 y uso de las TIC en la práctica docente. Comunicar, 37 (19), 169-175. doi: 10.3916/ C37-2011-03-09

Gallardo, E. (2012). Hablemos de estudiantes digitales y no de nativos digitales. UT. Revista de Ciències de l'Educació, (1), 7-21. Recuperado de http:// pedagogia.fcep.urv.cat/revistaut/revistes/ 2012juny/article01.pdf

González, J., Espuny, C. \& Gisbert, M. (2010). La evaluación cero de la competencia nuclear digital en los nuevos grados del EEES. Revista d'Innovació Educativa, 4, 1320.

González, P., De Pascual, A., Torres, E. \& Garnica, E. (2007). Una aplicación del análisis de componentes principales en el área educativa. Revista Economía,9, 55-72.

Jones, Ch., Ramanau, R., Cross, S. \& Healing, G. (2010). Net generation or digital natives: Is there a distinct new generation entering university?.Computers and Education, 54 (3), 722-732. doi: 10.1016/ j.compedu.2009.09.022

Karsenti, Th., Colin, S. \& Lira, M.L. (2012). Impacto del uso de los foros y los grupos de discusión en las prácticas pre-profesionales de los estudiantes de educación. Revista Apertura, 4(2). Recuperado dehttp:// www.udgvirtual.udg.mx/apertura/index.php/ apertura3/article/view/333/294

Kennedy, G., Judd, T., Churchwards, A., Gray, K.\& Krause, K.L. (2008). First year students' experiences with technology. Are they really digital natives?.Australasian 
Journal of Educational Technology, 24 (1), 108-122.

Krish, Pr. (2011).Fostering student engagement in online forums for language proficiency and knowledge enrichment. IPEDR, 5, 329-332.

Laurillard, D. (2002). Rethinking university teaching: A framework for the effective use of educational technology $\left(2^{\text {nd }}\right.$ ed.). London: Routledge.

Manprit, K. (2011). Using Online Forums in Language Learning and Education. Student Pulse Online Academic Journal, 3(3), 14.Recuperado de http://www.studentpulse. com/articles/414/using-online-forums-inlanguage-learning-and-education

Oblinger, D.G. \& Oblinger, J.L. (2005). Educating the net generation. Washington: EDUCAUSE. Recuperado de http:// www.educause.edu/educatingthenetgen/

O'Neill, P., Duplock, A. \& Willis, S. (2006). Using clinical experience in discussion with problem-based learning groups. Advances in HealthEducation, 11(4), 349-363.doi: 10.1007/s10459-006-9014-6

Palomar, M.J. (2009). Ventajas e inconvenientes de las TIC en la docencia. Revista digital inovación y experiencias educativas. $\mathrm{N}^{\mathrm{o}}$ de diciembre, 1-8. Recuperado de http://www.csi-csif.es/andalucia/modules/ mod_ense/revista/pdf/Numero_25/ MARIA_JOSE_PALOMAR_SANCHEZ01.pdf Passow, H.J. (2012). Which ABET competencies do engineering graduates find most important in their work?.Journal of Engineering Education, 101 (1), 95-118.doi: 10.1002/j.2168-9830.2012.tb00043.x

Peterson, C.L. \& Caverly, D.C.(2006). TechTalk: What students need to know about online discussion forums. Journal of Developmental Education, 29 (3),40-52.
Prensky, M. (2001). Digital Natives, Digital Immigrants. On the Horizon. MCB University Press, 9(5), 1-6. doi 10.1108/10748120110424816

Rosen, L.D. (2010). Rewired: Understanding the $i$-Generation and the way they learn. New York: Palgrave Macmillan.

Russo, T. \& Benson, S. (2005). Learning with Invisible Others: Perceptions of Online Presence and their Relationship to Cognitive and Affective Learning. Educational Technology and Society, 8 (1), 54-62.

Schuetze, U. (2010). Motivation to write online: Chats and Forums. German as a Foreign Language Journal, 1, 1-24. Recuperado de http://www.gfl-journal.de/12010/Schuetze.pdf

Tapscott, D. (1998). Growing Up Digital: The Rise of the Net Generation. New York: McGraw Hill.

Torres-Coronas, T. \& Vidal-Blasco, M.A. (2015). Students and employers perception about the development of digital skills in higher education. Revista de Educación, 367, 63-89.doi: 10.4438/1988-592X-RE-2015-367-283

Fecha de recepción: 12-02-2015

Fecha de evaluación: 12-04-2015

Fecha de aceptación: 30-04-2015 


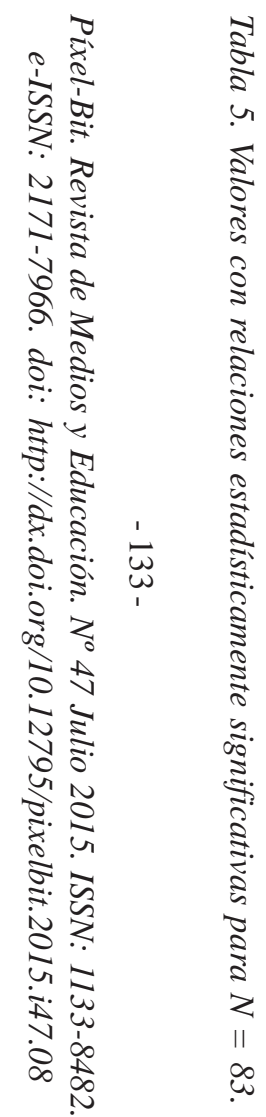

